\title{
Plasma transport and turbulence in the Helimak: Simulation and experiment
}

\author{
B. Li, ${ }^{1}$ B. N. Rogers, ${ }^{1}$ P. Ricci, ${ }^{2}$ and K. W. Gentle ${ }^{3}$ \\ ${ }^{1}$ Department of Physics and Astronomy, Dartmouth College, Hanover, New Hampshire 03755, USA \\ ${ }^{2}$ Centre de Recherches en Physique des Plasmas-École Polytechnique Fédérale de Lausanne, \\ Association EURATOM-Confédération Suisse, CH-1015 Lausanne, Switzerland \\ ${ }^{3}$ Department of Physics, Fusion Research Center, University of Texas at Austin, Austin, Texas 78712, USA
}

(Received 20 May 2009; accepted 4 August 2009; published online 25 August 2009)

\begin{abstract}
The Helimak experiment produces a toroidal plasma with a helical magnetic field. A simulation-experiment comparison of turbulence in this device is presented, focusing on parameter regimes in which the turbulence is dominated by interchange modes with $k_{\|} \simeq 0$. The numerical simulations are based on a two-dimensional electrostatic two-fluid model that evolves the full radial profiles of plasma density, the electric potential, and the electron temperature. The simulation results are compared with the experiment and general agreement is found for the plasma profiles, the autocorrelation functions, the frequency spectra, the cross-correlation functions, and the probability density functions. Some quantitative differences between the simulation and experimental data are also discussed. () 2009 American Institute of Physics. [DOI: 10.1063/1.3212591]
\end{abstract}

\section{INTRODUCTION}

The Helimak ${ }^{1,2}$ is a basic plasma physics experiment that produces a toroidal plasma with a helical magnetic field. The magnetic field lines wind around the symmetry axis of the torus and terminate on the upper and lower walls of the vessel, in rough analogy to the open field lines of the scrapeoff layer (SOL) of a tokamak. This magnetic configuration, sometimes called a "simple magnetized torus" or SMT, attracted significant experimental and theoretical interest, ${ }^{1-9}$ in part because it bears some similarity to the SOL region of tokamaks and also because it offers an analytically simple testbed in which to explore interchange and driftwave turbulence.

Past theoretical and experimental studies ${ }^{3,4}$ suggest the turbulence in the SMT configuration has two main drivers: interchange modes with $k_{\|}=0$ and driftwave instabilities with $k_{\|} \neq 0$. A key factor that controls the relative contributions of the two modes is thought to be the field line pitch. In the SMT, the vertical and toroidal magnetic fields combine to create a helical field with pitch $B_{v} / B_{\varphi}$. One toroidal circuit of $2 \pi R$ thus leads to a vertical displacement of the field lines given by $\Delta=2 \pi R B_{v} / B_{\varphi}=L_{v} / N$, where $R$ is the major radius, $L_{v}$ is the total vertical height of the chamber, and $N$ is the total number of toroidal turns made by a field line as it winds around the machine from bottom to top. The connection length, defined as the length of the open field lines, is therefore given by $L_{c}=L_{v} B_{\varphi} / B_{v}=2 \pi R N$. As we will show later, for the main case of interest here, an argon Helimak plasma with relatively short connection lengths and $N \sim 4$, the driftwave modes are strongly constrained by the longest parallel wavelengths $\lambda \sim L_{c}$ allowed in the system and have maximum growth rates that are much smaller than those of the interchange modes $\left(\gamma \sim c_{s} / \sqrt{R L_{p}}\right)$. This dominance of $k_{\|} \simeq 0$ interchangelike fluctuations motivates the field-line-averaged two-dimensional (2D) numerical model used in this paper. In the limit of long connection lengths (small $B_{v}$ ), on the other hand, the experiments, linear theory, as well as preliminary three-dimensional (3D) simulations all indicate that finite $k_{\|}$ modes become important. Future studies of the small $B_{v}$ regime will thus likely require 3D global simulations. The 2D model used here has simple boundary conditions in the vertical direction: since the fieldlines are displaced vertically by a distance $\Delta$ after one toroidal circuit, the simulated quantities, given $k_{\|}=0$, should be vertically periodic on this scale. In the simulations we thus consider a box of vertical height $\Delta$ with periodic vertical boundary conditions. These boundary conditions are only approximately correct, since the periodicity in the real system is eventually broken at the upper and lower walls of the plasma chamber. This discrepancy, as we discuss later, may represent one important source of physical error in the simulations.

A distinctive feature of an open fieldline system such as the SMT or tokamak SOL is the spontaneous formation of an equilibrium plasma potential $\phi_{0}$. This potential arises in the simulations due to the parallel boundary conditions at the sheath edge and is ultimately required to prevent the flux of electric charge from the plasma. For $\phi_{0}=0$ the outflow of electrons along the fieldlines to the walls would greatly exceed that of the ions due to their much larger thermal velocity. This imbalance would cause the plasma to become progressively more positively charged, which in turn would create a progressively larger electric field that would hold back the electron outflows and accelerate the ion outflows. At some point $\left[e \phi_{0} \simeq \Lambda T_{e 0}\right.$ in our system ${ }^{4}$ where $\Lambda=\ln \sqrt{m_{i} /\left(2 \pi m_{e}\right)}$ is the sheath parameter] the electric field becomes sufficient to cause the electron and ion outflows to be equal and the plasma potential therefore no longer increases. This equilibrium $\phi_{0}$ generates a vertical, sheared $\boldsymbol{E} \times \boldsymbol{B}$ flow $V_{E} \propto d \phi_{0} / d R \propto d T_{e} / d R$. In the parameter regime considered here, this flow has only a modest stabilizing impact on the turbulence. ${ }^{4}$ The associated $\boldsymbol{E} \times \boldsymbol{B}$ convection of the perturbations, however, leads to some of the most salient 
features of the statistical measures discussed in this paper: a vertical $\boldsymbol{E} \times \boldsymbol{B}$ flow $V_{E}$, coupled with vertical periodicity on the $\Delta$ scale, leads to temporal periodicity of the fluctuations at a given location on the timescale $\Delta / V_{E}$. This phenomenon is clearly reflected, for example, by a strong peak in the frequency spectra near $V_{E} / \Delta$.

This paper is organized as follows. In Sec. II, we review the derivation of the 2D fluid model from the 3D Braginskii equations. In Sec. III we explore the local linear stability of the model and calculate the local dispersion relations. In Sec. IV we describe the nonlinear simulations of the Helimak and calculate the equilibrium profiles and statistics of turbulence such as the sheared $\boldsymbol{E} \times \boldsymbol{B}$ flow, radial transport flux, frequency spectra, correlation functions, and probability distributions. The final section summarizes our findings.

\section{MODEL EQUATIONS}

In the Helimak, the electrons are preferentially heated by electron-cyclotron and upper-hybrid resonances and the electron-ion thermal equilibration time is much longer than a charge-exchange time and particle confinement times (for parallel loss), thus $T_{e} \gg T_{i}$ in the experiment. Typical plasma parameters for argon discharges are $\beta \sim 10^{-5}, B \sim 0.1 \mathrm{~T}$, $n \sim 10^{11} \mathrm{~cm}^{-3}, T_{i} \sim 0.1 \mathrm{eV}$, and $T_{e} \sim 10 \mathrm{eV}$. The toroidal chamber is rectangular with a radial extent $0.6 \mathrm{~m} \leq R$ $\leq 1.6 \mathrm{~m}$ and a vertical height of $L_{v}=2 \mathrm{~m}$. The plasma properties are measured by a large number of Langmuir probes located at the top and bottom plates. The probe arrays span $0.7 \mathrm{~m} \leq R \leq 1.5 \mathrm{~m}$ with spatial resolution of $1 \mathrm{~cm}$. For steep pitches, the measurements of profiles and fluctuations at the top and bottom are quite similar. The field strength in the Helimak is dominated by the vacuum toroidal field $B \simeq B_{0} R_{0} / R$ and the field line pitch may be varied up to $\Delta=1 \mathrm{~m}$.

The small $\beta$ and $T_{i}$ values in the experiment lead us to consider an electrostatic Braginskii numerical model with $T_{i} \simeq 0$. Neglecting for simplicity the dynamics associated with neutral particles, we therefore begin with the reduced equations $^{2,10-12}$ for the plasma density $n$, potential $\phi$, and electron temperature $T_{e}$ :

$$
\begin{aligned}
& d_{t} n=-\nabla \cdot\left(n V_{d e}\right)-n \nabla \cdot V_{E}-\nabla_{\|}\left(n V_{\| e}\right), \\
& \nabla \cdot\left(j_{d}+j_{p}\right)+\nabla_{\| j_{\|}}=0, \\
& \frac{3}{2} n d_{t} T_{e}=T_{e} d_{t} n+\frac{5}{2} \frac{p}{e}\left(\nabla \times \frac{\boldsymbol{b}}{B}\right) \cdot \nabla T_{e}+0.71 \frac{T_{e}}{e} \nabla_{\|} j_{\|},
\end{aligned}
$$

where $d_{t}=\partial_{t}+V_{E} \cdot \nabla$ is the convective derivative, $\boldsymbol{V}_{E}=(\boldsymbol{b} / B)$ $\times \nabla \phi$ is the $\boldsymbol{E} \times \boldsymbol{B}$ velocity, $p \simeq n T_{e}$ is the plasma pressure, $\boldsymbol{j}_{d}=(\boldsymbol{b} / B) \times \nabla p$ is the diamagnetic current, $\boldsymbol{V}_{d e} \simeq-\boldsymbol{j}_{d} /$ en is the electron diamagnetic velocity, $\boldsymbol{j}_{p}=\left(-n m_{i} / B^{2}\right) d_{t} \nabla \boldsymbol{\phi}$ is the polarization current, $j_{\|}=e n\left(V_{\| i}-V_{\| e}\right)$ is the parallel current, and $V_{\| e}$ and $V_{\| i}$ are the electron and ion parallel velocities. The curvature operator is defined as

$$
\hat{C}=\left(\nabla \times \frac{b}{B}\right) \cdot \nabla
$$

and for $\nabla \times(B \boldsymbol{b}) \simeq 0$ with negligible local current density, $\nabla \times(\boldsymbol{b} / B)=(\boldsymbol{b} \times \nabla B) / B^{2}+(\boldsymbol{b} \times \boldsymbol{\kappa}) / B=2(\boldsymbol{b} \times \boldsymbol{\kappa}) / B, \quad$ where $\boldsymbol{\kappa}=(\boldsymbol{b} \cdot \nabla) \boldsymbol{b}$ is the magnetic curvature. In the presence of magnetic curvature, one obtains $\nabla \cdot V_{E}=\hat{C} \phi$ and $\nabla \cdot j_{d}=\hat{C} p$. Here the divergence of polarization current is approximated as ${ }^{2,4}$ $\nabla \cdot \boldsymbol{j}_{p} \approx\left(-n m_{i} / B^{2}\right) d_{t} \nabla^{2} \phi$. For numerical simplicity this expression neglects some terms associated with the density variations (the Boussinesq approximation). This approximation represents another possible source of physical error in the simulations, particularly at the largest $R$ values where the relative density fluctuations in the nonlinear state become large.

We now consider field-aligned coordinates in which ${ }^{4} \hat{z}$ is the direction along the magnetic field, $\hat{x}=\hat{R}$ is the radial direction, and $\hat{y}$ is perpendicular to both $\hat{x}$ and $\hat{z}$. For a strong toroidal field, $\hat{y}$ is nearly in the vertical (poloidal) direction. Specializing on the case of interchange modes, we assume that the density, temperature, and potential are approximately constant along the field lines in the main plasma. Following standard sheath theory, ${ }^{13}$ the ion and electron fluxes at the ends of open field lines are taken as ${ }^{4} \int_{0}^{L_{c}} \nabla_{\|}\left(n V_{\| i}\right) d z=n c_{s}$ and $\int_{0}^{L_{c}} \nabla_{\|}\left(n V_{\| e}\right) d z=n c_{s} \exp \left(\Lambda-e \phi / T_{e}\right)$, respectively, where $\Lambda$ $=\ln \sqrt{m_{i} /\left(2 \pi m_{e}\right)} \simeq 4.7$ is the sheath parameter for argon and $c_{s}=\sqrt{T_{e} / m_{i}}$ is the sound speed. Integrating Eqs. (1)-(3) along the parallel direction $\int_{0}^{L_{c}} d z / L_{c}$ leads to the field-lineaveraged $2 \mathrm{D}$ equations for plasma density $n(x, y, t)$, potential $\phi(x, y, t)$, and electron temperature $T_{e}(x, y, t)$ :

$$
\begin{aligned}
& d_{t} n= \frac{\hat{C} p}{e}-n \hat{C} \phi-n \frac{c_{s}}{L_{c}} \exp \left(\Lambda-e \phi / T_{e}\right), \\
& \frac{n m_{i}}{B^{2}} d_{t} \nabla^{2} \phi=\hat{C} p-e n \frac{c_{s}}{L_{c}}\left[\exp \left(\Lambda-e \phi / T_{e}\right)-1\right], \\
& d_{t} T_{e}=\frac{2}{3} T_{e}\left(\frac{\hat{C} p}{e n}-\hat{C} \phi+\frac{5}{2} \frac{\hat{C} T_{e}}{e}\right) \\
& \quad-\frac{2}{3} T_{e} \frac{c_{s}}{L_{c}}\left[1.71 \exp \left(\Lambda-e \phi / T_{e}\right)-0.71\right] .
\end{aligned}
$$

For consistency with the Helimak experiment, the magnetic field direction is $\boldsymbol{b}=-\hat{z}$. Thus $\boldsymbol{\kappa}=-\hat{x} / R, \hat{C}=(2 / B R) \partial_{y}$, and $\boldsymbol{V}_{E} \cdot \nabla=\left(\partial_{y} \phi \partial_{x}-\partial_{x} \phi \partial_{y}\right) / B$.

In the simulations we normalize $n$ to $\mathcal{N}, B$ to $B_{0}, T_{e}$ and $e \phi$ to $\mathcal{T}$, time to $t_{0}=R_{0} / c_{s}$, and length to $\rho_{s}=c_{s} / \Omega_{i}$, where $\mathcal{N}$ and $\mathcal{T}$ are fixed reference values, $c_{s}=\sqrt{\mathcal{T} / m_{i}}$, and $\Omega_{i}=e B_{0} / m_{i}$. To convert the normalized simulation results to physical values, we use the following nominal values of argon discharges in the Helimak: ${ }^{2} \mathcal{T}=10 \mathrm{eV}, c_{s}=5$ $\times 10^{3} \mathrm{~m} / \mathrm{s}, \rho_{s}=2 \mathrm{~cm}, R_{0}=1.1 \mathrm{~m}$, and $t_{0} \simeq 0.22 \mathrm{~ms}$. To model the plasma production and heating in the experiment, source terms $S_{n}$ and $S_{T}$ are added to the density and temperature equations. Including the source and diffusion terms, Eqs. (4)-(6) in normalized form become 


$$
\begin{aligned}
d_{t} n= & 2 n\left(\partial_{y} T_{e}+\frac{T_{e}}{n} \partial_{y} n-\partial_{y} \phi\right)+\nu_{n} \nabla^{2} n \\
& -\sigma n \sqrt{T_{e}} \exp \left(\Lambda-\phi / T_{e}\right)+S_{n}, \\
d_{t} \nabla^{2} \phi= & 2 B^{2}\left(\partial_{y} T_{e}+\frac{T_{e}}{n} \partial_{y} n\right)+\nu_{\phi} \nabla^{4} \phi \\
& -\sigma \sqrt{T_{e}}\left[\exp \left(\Lambda-\phi / T_{e}\right)-1\right] \\
d_{t} T_{e}= & \frac{4}{3} T_{e}\left(\frac{7}{2} \partial_{y} T_{e}+\frac{T_{e}}{n} \partial_{y} n-\partial_{y} \phi\right)-\frac{2}{3} \sigma T_{e}^{3 / 2} \\
& \times\left[1.71 \exp \left(\Lambda-\phi / T_{e}\right)-0.71\right]+\nu_{T} \nabla^{2} T_{e}+S_{T},
\end{aligned}
$$

where $d_{t}=\partial_{t}+\left(R_{0} / \rho_{s}\right)\left(\partial_{y} \phi \partial_{x}-\partial_{x} \phi \partial_{y}\right) / B$ indicates the convective derivative and $\sigma=R_{0} / L_{c}$. The model Eqs. (7)-(9) are very similar to those used previously to study interchange dynamics in other systems. ${ }^{4,14-16}$ The normalized diffusion constants are taken to be $\nu_{n}=\nu_{t}=1$ and $\nu_{\phi}=0.5$. Localized source profiles with Gaussian shapes are used for density and temperature in the simulations; ${ }^{4}$ that is, the shapes of the sources are given by $S_{n}=S_{T}=S_{0} \exp \left[-\left(x-x_{s}\right)^{2} / w^{2}\right]$. The location, width, and strength of the effective sources are chosen so that the simulations approximately reproduce the shape and magnitude of the profile peaks measured in the experiments. The profiles used are also reasonable representations of the power absorbed by the combination of the electron cyclotron resonance and the upper hybrid resonance, which is effectively broadened by the large density fluctuations. ${ }^{1,17}$ Here the strength and width of the sources are taken to be $S_{0}=0.1$ and $w=5$, and $S_{n}$ and $S_{T}$ are centered at $R=1 \mathrm{~m}$ and $R=1.1 \mathrm{~m}$, respectively. As noted earlier we consider a radially global simulation domain $R=0.6 \mathrm{~m}$ to $R=1.6 \mathrm{~m}$ with Dirichelet boundary conditions in the radial direction and a vertical box size $L_{y}=\Delta$ with periodic boundary conditions in the $y$ direction. Thus $k_{\Delta}=2 \pi / \Delta$ is the lowest $k_{y}$ mode in the simulation. In the experiment, quantities such as $L_{c} \propto B_{\varphi}$ $\propto 1 / R, \sigma \propto 1 / L_{c} \propto R$, and $\Delta \propto R / B_{\varphi} \propto R^{2}$ have significant radial variation across the chamber. For simplicity in the simulations, however, we evaluate these quantities at the middle of the vessel $R=1.1 \mathrm{~m}$, corresponding to the choices $\sigma=0.04, \Delta=2 \pi L_{v} \sigma \simeq 0.5 \mathrm{~m}, \quad L_{c} \simeq 28 \mathrm{~m}, \quad B_{v} / B_{\varphi}=L_{v} / L_{c}$ $\simeq 0.07, N=L_{v} / \Delta \simeq 4$, and $\rho_{s} k_{\Delta} \simeq 0.25$. This approximation is convenient in the simulations since $\Delta$, the vertical box height, is a constant. As we will discuss later, however, it represents yet another source of potential physical error in the simulations.

\section{LINEAR ANALYSIS}

In this section we analyze the local linear stability of the model. Quantities such as the density are expressed as $n=n_{0}+\tilde{n}$, where $n_{0}$ and $\tilde{n}$ represent background profiles and perturbations, respectively. It is convenient to introduce the gradient scale lengths $L_{n}=-n_{0} / n_{0}^{\prime}, L_{T e}=-T_{e 0} / T_{e 0}^{\prime}$, and $L_{\phi}=-\phi_{0} / \phi_{0}^{\prime}$, where primes denote normalized radial derivatives $d / d x$, and the frequencies $\omega_{*}=k_{y} \rho_{s} c_{s} / L_{n}, \omega_{\kappa}$ $=2 k_{y} \rho_{s} c_{s} / R=2 \omega_{*} L_{n} / R$, and $\omega_{E}=k_{y} V_{E}$, where $\rho_{s} c_{s}=T_{e 0} / e B$ and $V_{E}=-\phi_{0}^{\prime} / B$ is the background poloidal $\boldsymbol{E} \times \boldsymbol{B}$ flow. Here we normalize the perturbations $\tilde{n}, \widetilde{T}_{e}$, and $\widetilde{\phi}$ to the local background values $n_{0}, T_{e 0}$, and $T_{e 0} / e$, respectively, and assume the perturbations are proportional to $\exp \left(i k_{y} y-i \omega t\right)$, where $\omega=\omega_{r}+i \gamma$ is complex, $\omega_{r}$ is the frequency in the laboratory frame, and $\gamma$ is the local linear growth rate. Linearizing Eqs. (4)-(6) we find

$$
\begin{aligned}
& -\bar{\omega} \tilde{n}-\omega_{*} \tilde{\phi}=\omega_{\kappa}(\tilde{T}+\tilde{n}-\tilde{\phi})-\frac{\sigma}{t_{0}}\left[\tilde{n}+\left(\Lambda+\frac{1}{2}\right) \tilde{T}-\tilde{\phi}\right], \\
& \bar{\omega} k_{y}^{2} \rho_{s}^{2} \widetilde{\phi}=\omega_{\kappa}(\widetilde{T}+\tilde{n})-\frac{\sigma}{t_{0}}(\Lambda \widetilde{T}-\widetilde{\phi}), \\
& -\bar{\omega} \tilde{T}-\eta \omega_{*} \widetilde{\phi}=\frac{2}{3} \omega_{\kappa}\left(\frac{7}{2} \tilde{T}+\tilde{n}-\tilde{\phi}\right) \\
& -\frac{2}{3} \frac{\sigma}{t_{0}}\left[\frac{3}{2} \widetilde{T}+1.71(\Lambda \widetilde{T}-\widetilde{\phi})\right]
\end{aligned}
$$

where $\bar{\omega}=\omega-\omega_{E}$ is the frequency in the local plasma rest frame, $\eta=L_{n} / L_{T e}, \sigma=R / L_{c}$, and $t_{0}=R / c_{s}$. The sheath terms $\exp \left[\left(\Lambda T_{e}-e \phi\right) / T_{e}\right]$ in Eqs. (4)-(6) have been linearized as $1+\Lambda \widetilde{T}-\widetilde{\phi}$, since $e \phi \approx \Lambda T_{e}$ as shown in the nonlinear simulations to be discussed in the next section. For $k_{y} \rho_{s} \ll 1$ and $\gamma \gg \omega_{\kappa}$, Eqs. (10) $-(12)$ with $\sigma=0$ gives

$$
\begin{aligned}
& \bar{\omega} \widetilde{p}+\omega_{*}(1+\eta) \widetilde{\phi}=\frac{5}{3} \omega_{\kappa} \widetilde{\phi}, \\
& \bar{\omega} k_{y}^{2} \rho_{s}^{2} \widetilde{\phi}=\omega_{\kappa} \widetilde{p},
\end{aligned}
$$

where $\tilde{p}$ is normalized to the background pressure $p_{0}$. Equations (13) and (14) are the linearized equations for $d_{t} p=-(5 / 3) p \hat{C} \phi$ and $\left(n m_{i} / B^{2}\right) d_{t} \nabla^{2} \phi=\hat{C} p$ and give rise to the interchange mode

$$
-\bar{\omega}^{2}=\frac{2 c_{s}^{2}}{R L_{p}}\left(1-\frac{10}{3} \frac{L_{p}}{R}\right),
$$

where $L_{p}=-p_{0} / p_{0}^{\prime}=L_{n} /(1+\eta)$ is the pressure gradient scalelength. For sufficiently steep pressure gradients $\left(L_{p} / R<0.3\right)$ in the bad-curvature region, where $L_{p}>0$, Eq. (15) gives the interchange growth rate $\gamma_{0} \simeq \sqrt{2} c_{s} / \sqrt{R L_{p}}$.

Figure 1 shows the local dispersion relation obtained from Eqs. (10)-(12) for the parameters relevant to the simulations. Here $L_{n} / R=0.23, \eta=1, \sigma=0.04, \Lambda=4.7$, and $e \phi_{0} \simeq \Lambda T_{e 0}$, with $L_{T e} \simeq L_{\phi}$. As shown in Fig. 1(a), the lowest $k_{y}$ mode allowed in the system $\rho_{s} k_{\Delta} \simeq 0.25$ corresponds to the fastest growing instability with a growth rate comparable to the interchange growth rate $\gamma \sim \gamma_{0}$. For $k_{y} \rightarrow 0$, the linear growth $\gamma$ is reduced below the interchange growth rate because of the sheath effects. Figure 1(b) shows the frequency of the instability in the local plasma rest frame $\bar{\omega}=\omega_{r}-\omega_{E}$. In the laboratory frame, the frequency of the instability is shifted by the $\boldsymbol{E} \times \boldsymbol{B}$ frequency $\omega_{E}$. When the background $\boldsymbol{E} \times \boldsymbol{B}$ flow is large, the dispersion relation measured in the laboratory frame is close to $\omega_{E}=k_{y} V_{E}$ with a linear structure 

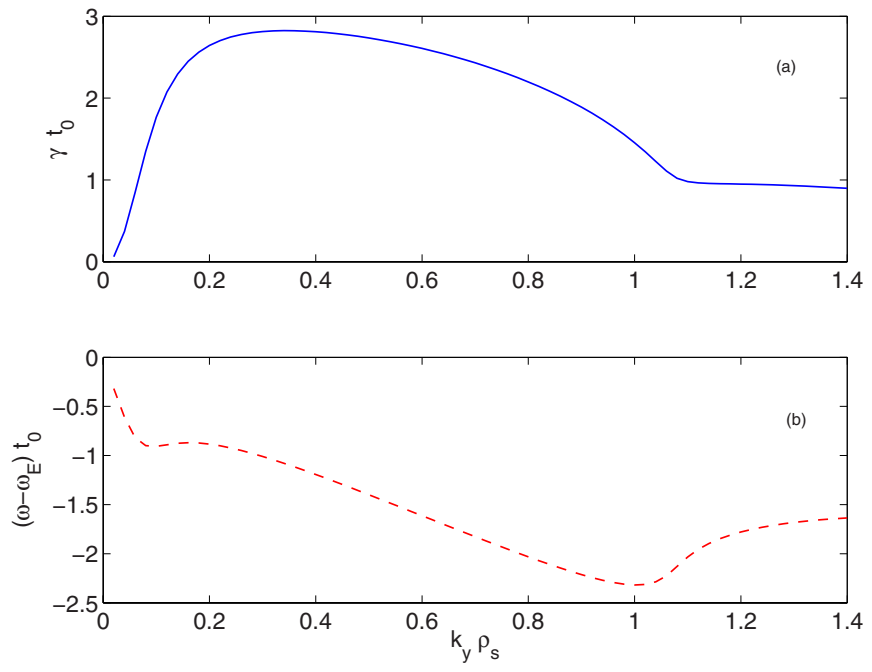

FIG. 1. (Color online) Typical normalized growth rate $\gamma t_{0}$ with $t_{0}=R / c_{s}$ and frequency $\bar{\omega} t_{0}$ of an interchange instability.

and the phase velocity of the perturbations in the vertical direction is therefore similar to the background $\boldsymbol{E} \times \boldsymbol{B}$ flow.

Although sheath effects introduce a low- $k_{y}$ cutoff in the interchange mode growth rate, the combination of the sheath terms and the temperature gradient $T_{e}^{\prime}$ can also introduce an instability into the system. ${ }^{18}$ For our parameters, this instability seems to be overpowered by the interchange mode. This has been confirmed by nonlinear simulations of our model without the magnetic curvature, in which only the sheath mode and possibly Kelvin-Helmholtz modes are present. The resulting mode activity and transport are extremely weak compared to the levels driven by the interchange mode to be discussed in the next section.

As noted earlier, the application of the 2D model used here requires that driftwave modes are much weaker than interchange instabilities. The validity of this for our parameters can be tested with the dispersion relation of a corresponding 3D model [see Eq. (5) in Ref. 3]. For the parameters of argon discharge studied here $\left(m_{e} / m_{i} \simeq 10^{-5}\right.$, $L_{n} / R=0.23, \quad \eta=1, \quad$ minimum parallel wave number $\left.k_{\|} R=\pi R / L_{c} \simeq 0.1, \nu_{e i} t_{0} m_{e} / m_{i} \sim 10^{-4}\right)$ the driftwave instabilities driven by resistivity and electron inertia have maximum growth rates $\gamma t_{0} \simeq 0.1$ at $k_{y} \rho_{s} \simeq 1$ that are much smaller than the interchange modes in our system. As a further check, we also carried out a small number of 3D nonlinear global simulations of the system described here and, in the short connection length (large vertical field) limit, we find essentially the same turbulence and transport levels as in the 2D model. On the other hand, at small vertical fields, deviations from the 2D model appear in both the 3D simulations, linear theory, ${ }^{3}$ and the experiments, in which (for example) the asymmetries of profiles measured at the top and bottom of the chamber become noticeable for lower pitches. Thus the validity of the 2D model may be restricted to the limit that is studied in the paper (short connection lengths or large vertical fields). The 3D work will be described in detail in a future publication.
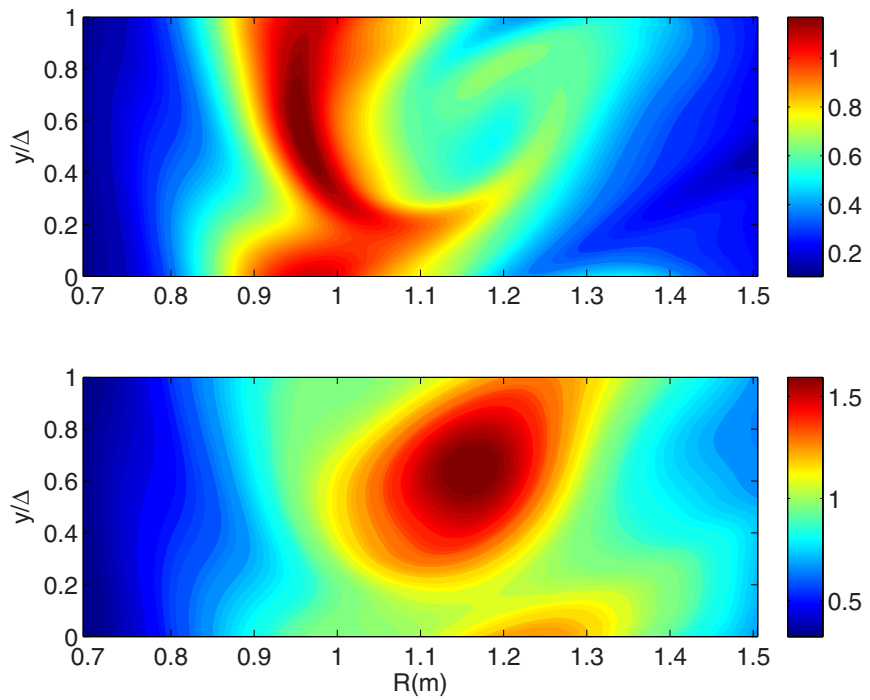

FIG. 2. (Color online) Typical spatial structures of plasma pressure $p / \mathcal{N T}$ (upper plot) and plasma potential $e \phi / \Lambda \mathcal{T}$ (lower plot).

\section{NONLINEAR REGIME: SIMULATIONS AND EXPERIMENT}

In this section, we compare nonlinear simulations of the Helimak to the experiments for short connection lengths $L_{c} \sim 20 \mathrm{~m}$. The nonlinear Eqs. (7)-(9) are solved with a numerical code developed from the $2 \mathrm{D}$ advection-diffusion solver ${ }^{14,19}$ that implements the algorithm described in Ref. 20. The simulations are started from small amplitude random perturbations with uniform backgrounds for all fields. The source terms in the simulations initially produce steep plasma profiles that are strongly unstable to interchange modes. These instabilities produce turbulent cross-field transport and a relaxation of the average gradients, until eventually a turbulent, statistically steady state is reached in which the production of the plasma by the sources is balanced by the parallel losses. The total time of simulation is $t=8000 t_{0}$ to ensure the convergence of results. Figure 2 shows typical snapshots of the plasma pressure and potential in the quasisteady, turbulent nonlinear phase. Consistent with expectations based on linear theory, the lowest $k_{y}=k_{\Delta}$ mode is the dominant instability. The contours of the plasma potential exhibit large-scale $\boldsymbol{E} \times \boldsymbol{B}$ eddies in the bad-curvature region. Such eddies lead to a radial mixing of plasma, a flattening of the plasma gradients, and ultimately a saturation of the unstable modes.

Figure 3 shows the time-averaged profiles of plasma density $n_{0} / \mathcal{N}$, electron temperature $T_{e 0} / \mathcal{T}$, and plasma potential $e \phi_{0} / \Lambda \mathcal{T}$. The potential roughly follows the temperature with $\phi_{0} \sim \Lambda T_{e 0}$ due to the sheath boundary conditions. The peak of $\phi_{0}$ produces a peak in the flow shear $\propto \phi_{0}^{\prime \prime}$, which in turn leads to a modest steepening of the density profile. The typical density and temperature profiles in the experiments are shown in Fig. 4 ( $\phi$ is discussed below). The experimental profiles exhibit a radial offset between the density and temperature profiles that is reproduced in the simulations by the placement of the density and temperature sources. The experimental profiles are seen to drop somewhat more steeply with increasing $R$ than the simulations. In the bad-curvature 


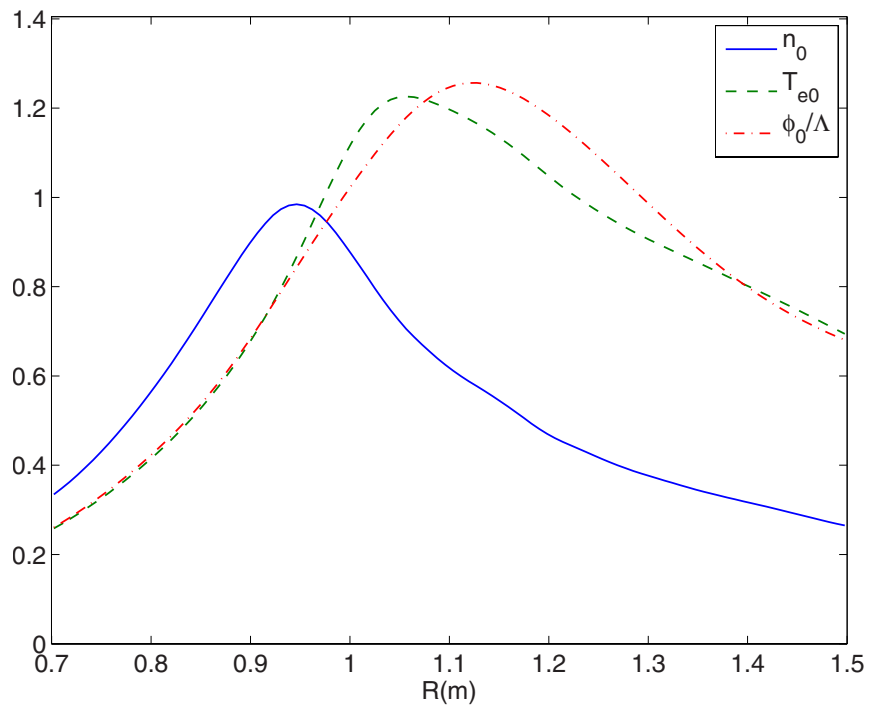

FIG. 3. (Color online) Typical profiles of plasma density $n_{0} / \mathcal{N}$, electron temperature $T_{e 0} / \mathcal{T}$, and plasma potential $e \phi_{0} / \Lambda \mathcal{T}$. Here $\Lambda=4.7$ for argon discharges.

region, the pressure gradient scalelength $L_{p} \approx 0.18 \mathrm{~m}$ in the experiment and $L_{p} \simeq 13 \rho_{s} \approx 0.26 \mathrm{~m}$ in the simulation. Reference 8 shows that $L_{p}$ in this interchange dominated system is determined by the expression $\sqrt{2}\left(R / L_{p}-10 / 3\right)^{3 / 2} \simeq 4 R / L_{v}$. For the Helimak device $L_{v} / R \approx 2$, this yields $L_{p} / R \approx 0.22$, which generally agrees with the simulation. The larger profile scalelengths in the simulations may be due to the neglect of the radial variation of $\sigma \propto R$, which in the experiments leads to a relative strengthening of the parallel losses with increasing $R$. This strengthening is due to the reduction in $B_{\varphi} \propto 1 / R$ with $R$, which in turn increases in the fieldline pitch and thus the component of the (sonic) parallel flows that are perpendicular to the walls. The cross-field transport at large $R$ may also be enhanced relative to the simulations by the radial increase in $\Delta$, which allows longer vertical wavelength

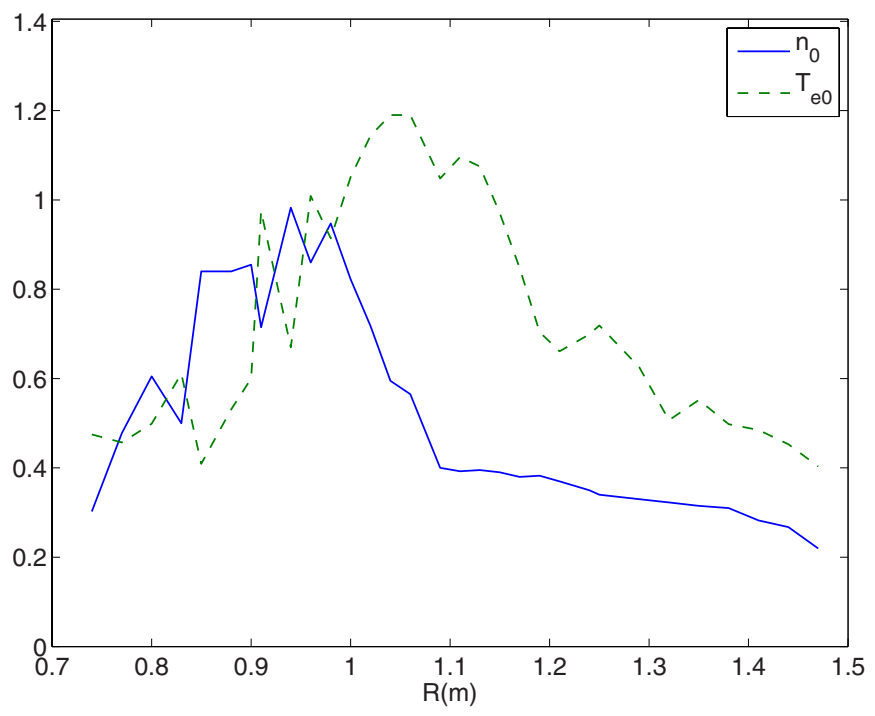

FIG. 4. (Color online) Typical experimental profiles of plasma density $n_{0} / \mathcal{N}$ and electron temperature $T_{e 0} / \mathcal{T}$. Here $\mathcal{N}=4 \times 10^{10} \mathrm{~cm}^{-3}$ and $\mathcal{T}=10 \mathrm{eV}$.

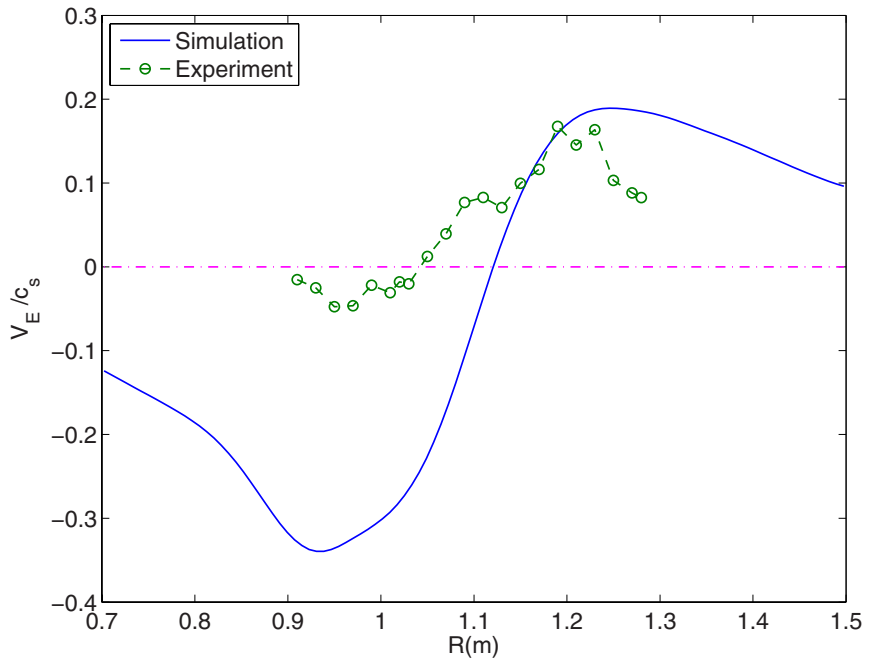

FIG. 5. (Color online) Typical profiles of equilibrium poloidal $\boldsymbol{E} \times \boldsymbol{B}$ flow $V_{E} / c_{s}$ in the simulation (solid line) and the measured flow in the experiment (circles).

instabilities into the system. Neither of these effects are included in the simulations, in which radial variation of the pitch and box size $\Delta$ are neglected.

The plasma potential $\phi$ in the experiments may be estimated from Langmuir probe measurements of the floating potential $V_{f}$ as $^{21} \phi \approx V_{f}+\Lambda T_{e} / e$ and is found to be, with large error bars, qualitatively similar to that in Fig. 3. Alternatively, as shown in Fig. 5, one can compare typical profiles of the poloidal $\boldsymbol{E} \times \boldsymbol{B}$ flow $V_{E} / c_{s}=E_{r} / B_{0} c_{s}=-\phi_{0}^{\prime}$ from the simulation with the measured flow in the experiment, normalized to the nominal sound speed $c_{s} \approx 5 \times 10^{3} \mathrm{~m} / \mathrm{s}$. The Doppler spectroscopy measurement of the flow is a true average over the full height of the device; thus the parallel flows, for example, which are in opposite directions at the top and bottom of the chamber, average out of the measurements. The experimental velocity data have a large systematic uncertainty of about $0.2 c_{s} \approx 10^{3} \mathrm{~m} / \mathrm{s}$. Despite this, however, the experimental flows in the downward direction (negative $y$ ) seem to be weaker than the simulations on the high field side. As a consequence, the slope of the flow profile, which is the $\boldsymbol{E} \times \boldsymbol{B}$ shear $d V_{E} / d R$ is weaker in the experiment than in the simulation. The stronger flows in the simulation may be an artifact of the periodic vertical boundary conditions, which allow a continuous recirculation of the vertical zonal flows-recirculation that is ultimately inhibited in the experiment by the upper and lower chamber walls. Global 3D simulations are in progress, which will include more realistic boundary conditions.

Figure 6 shows the time-averaged profiles of pressure $p_{0}=n_{0} T_{e 0}$, normalized to $\mathcal{N} \mathcal{T}$, and the flow shear rate $V_{E}^{\prime} / \Omega_{i}=-\phi_{0}^{\prime \prime}$, normalized to the linear growth rate of interchange instability $\gamma_{0} t_{0} \simeq \sqrt{2 R / L_{p}} \approx 3$. As seen in Fig. 6 , the peak shear rate is comparable to the linear growth rate. In contrast with studies of microturbulence that often find strong stabilizing effects at such $\boldsymbol{E} \times \boldsymbol{B}$ flow levels, our previous investigations of this system ${ }^{4}$ found that the background $\boldsymbol{E} \times \boldsymbol{B}$ flow in this system becomes important to the transport only at a somewhat higher level: $V_{E}^{\prime} / \gamma_{0} \simeq 2$. This is 


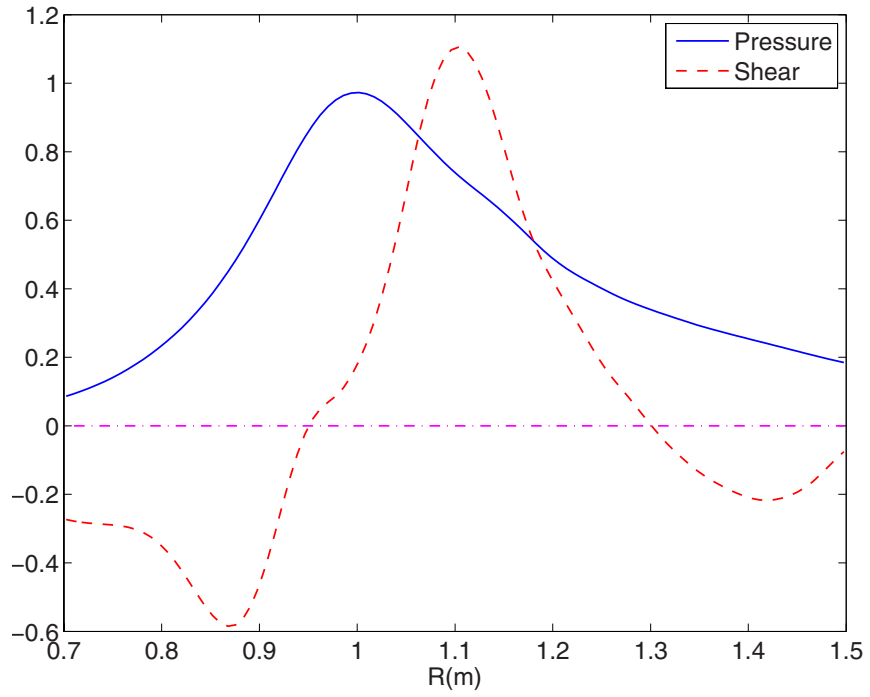

FIG. 6. (Color online) Typical profiles of plasma pressure $p_{0} / \mathcal{N T}$ and $\boldsymbol{E} \times \boldsymbol{B}$ flow shear $V_{E}^{\prime} / \gamma_{0}$.

possibly due to the radially global nature of the instabilities in the present system, which can more effectively reach across the $\boldsymbol{E} \times \boldsymbol{B}$ shear layers.

Figure 7 shows the time-averaged profiles of the radial particle flux generated by the turbulence, defined as $\Gamma / \mathcal{N} c_{s}=\left\langle\tilde{n} \partial_{y} \widetilde{\phi}\right\rangle$, where $\partial_{y} \widetilde{\phi}$ represents the fluctuating radial velocity and $\langle\cdots\rangle$ indicates averages over time and the vertical direction $y$. The potential fluctuations produce the fluctuations in the vertical electric field $E_{y}=-\partial_{y} \widetilde{\phi}$, which leads to the fluctuating radial $\boldsymbol{E} \times \boldsymbol{B}$ velocity. The mean radial $\boldsymbol{E} \times \boldsymbol{B}$ flow is zero, since $\left\langle E_{y}\right\rangle=0$. Neglecting the (small) diffusion terms, averaging Eq. (7) over time and the vertical direction yields

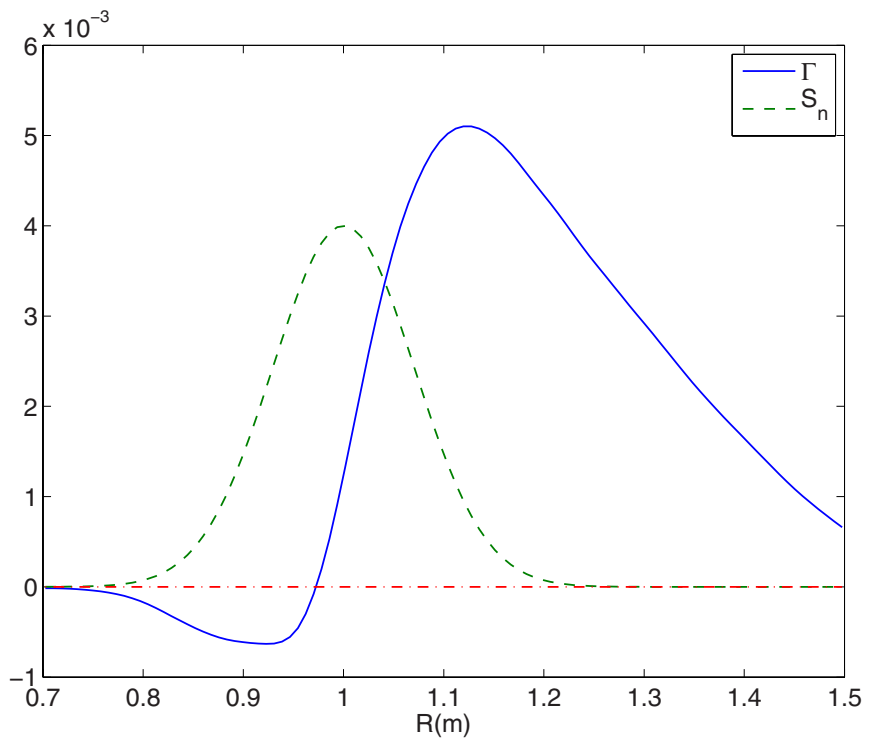

FIG. 7. (Color online) Typical profile of radial particle flux $\Gamma / \mathcal{N} c_{s}$ and the profile of density source $S_{n}$ (arbitrary units).

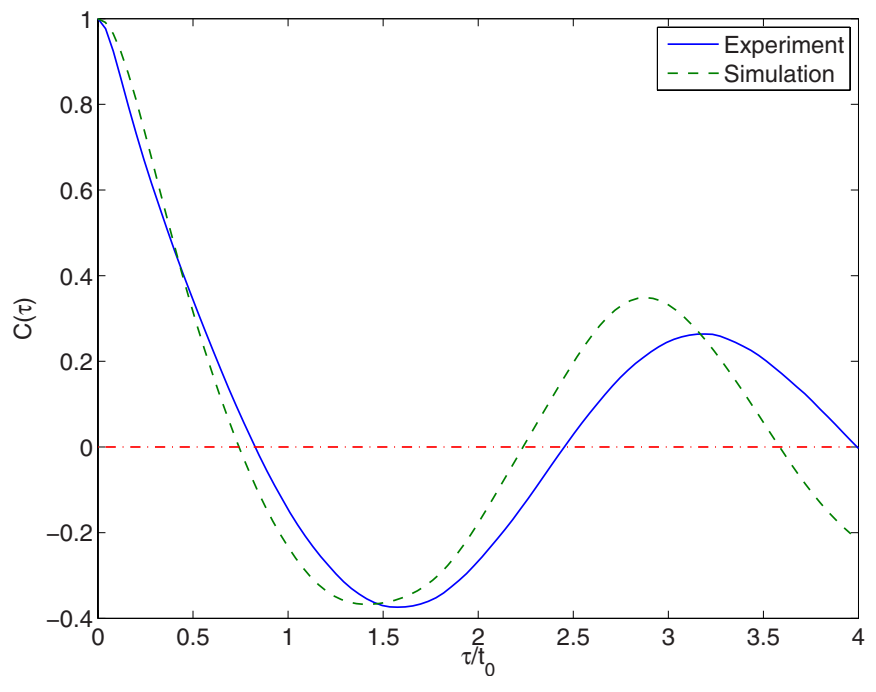

FIG. 8. (Color online) Comparison of experimental data (solid line) and simulation (dashed line) for the typical autocorrelation function of fluctuations. Here $t_{0} \simeq 0.22 \mathrm{~ms}$.

$$
\frac{R_{0}}{\rho_{s}} \frac{d \Gamma}{d x} \simeq S_{n}-\sigma n_{0} \sqrt{T_{e 0}} .
$$

Equation (16) shows that the plasma source $\left(S_{n}\right)$, parallel losses $(\propto \sigma)$, and the radial transport $(\Gamma)$ produced by the turbulence determine the equilibrium profiles. As seen in Fig. 7, the outward (positive) flux increases sharply and reaches a maximum near the source peak, which is centered at $R=1 \mathrm{~m}$ with a width of $0.1 \mathrm{~m}$, and decreases slowly thereafter. Parametrizing the turbulent flux in terms of an effective diffusion coefficient $D$ as $\Gamma=-D n_{0}^{\prime}$, we obtain a maximum value $D \approx 0.2 \rho_{s} c_{s}$ near the peak flux. Reference 8 shows that the diffusion coefficient of interchange mode in this system is given by $D \simeq 0.34 \sqrt{2 L_{p} / R}\left(1-10 L_{p} / 3 R\right)^{3 / 2} c_{s} / k_{\Delta} \approx 0.17 \rho_{s} c_{s}$ for $L_{p} / R \approx 0.2$, which generally agrees with the simulation.

We now turn to a more quantitative study of the density fluctuations in the turbulent state for short connection lengths $L_{c} \sim 20 \mathrm{~m}$. For simplicity we focus on the statistical properties of the turbulence at a typical radial location on the low field side $(R \approx 1.2 \mathrm{~m})$ where the turbulence is strong. In the experiment the density fluctuations are measured from Langmuir probes collecting ion saturation current and the details of experimental measurements are described in Ref. 2. In the following we will focus on four common statistical measures of the fluctuations: the autocorrelation function, the frequency spectrum, the cross-correlation function, and the probability density function (PDF). All of these measures show reasonably good agreement between the experiment and simulation. A range of other radial locations in the badcurvature region where $d p / d R<0$ yield qualitatively similar results.

Figure 8 shows the experimental and simulation data of the typical autocorrelation function of the fluctuations, defined as $C(\tau)=\langle\widetilde{n}(t) \widetilde{n}(t+\tau)\rangle / \sigma^{2}$, where $\langle\cdots\rangle$ indicates time average, $\tau$ denotes the time difference, and $\sigma^{2}=\left\langle\widetilde{n}^{2}(t)\right\rangle$ is the mean square value of fluctuations. The oscillation of correlations in Fig. 8 has a dominant frequency $f \approx 0.3 / t_{0}$, which is about the $\boldsymbol{E} \times \boldsymbol{B}$ frequency $\omega_{E} / 2 \pi=V_{E} / \Delta \approx 0.4 / t_{0}$ with 


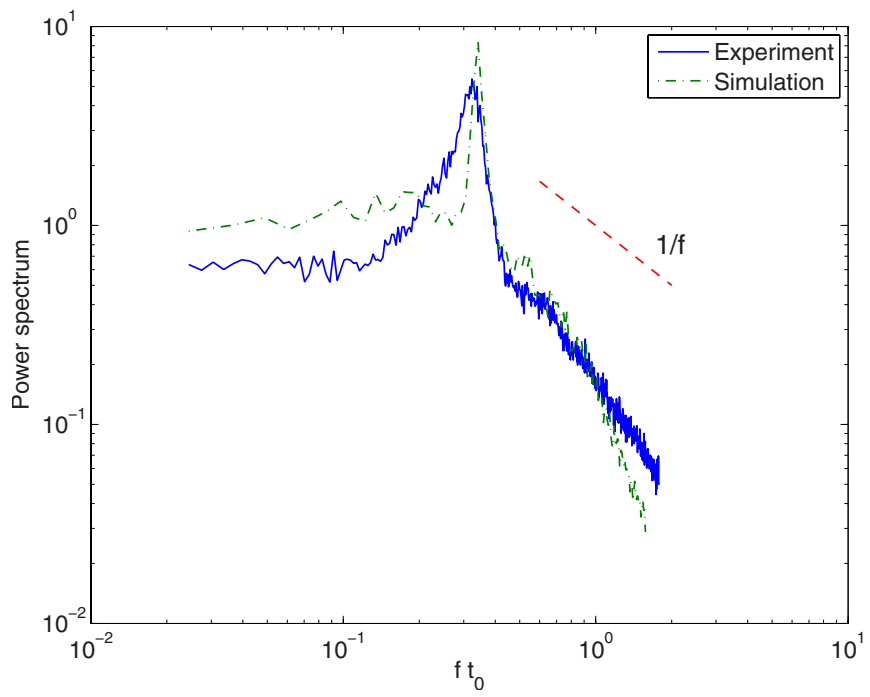

FIG. 9. (Color online) Comparison of experimental data (solid line) and simulation (dashed line) for the typical power spectrum of fluctuations. Here $1 / t_{0} \simeq 4.5 \mathrm{kHz}$.

$V_{E} \approx 0.2 c_{s}$ at $R \simeq 1.2 \mathrm{~m}$. Thus, the oscillation frequency of the correlation functions are very close to the frequency of the dominant $k_{y}=k_{\Delta}$ instability in the laboratory frame. For oscillating autocorrelations like those of Fig. 8, one has to make some estimate of the envelope of the oscillation to infer the decorrelation time ( $\sim 1 / e$ decay time). In Fig. 8 , the first negative maximum at $\tau \simeq 1.5 t_{0} \approx 0.3 \mathrm{~ms}$ happens at a value $\sim 1 / e$ and thus seems a good estimate of the decorrelation time.

Figure 9 shows the experimental and simulation data of a typical power spectral density function of fluctuations. This function is defined as $\left\langle|\widetilde{n}(f)|^{2}\right\rangle / \sigma^{2} \Delta f$, where $\langle\cdots\rangle$ indicates ensemble average, $\widetilde{n}(f)$ represents the Fourier transform of $\tilde{n}(t)$, the total power $\sigma^{2}$ equals the sum of $|\tilde{n}(f)|^{2}$ over all frequencies, and $\Delta f$ is the frequency resolution. As seen in Fig. 9, the observed power spectrum has a narrow peak at low frequencies and becomes broad at high frequencies. The slow decay of the power spectra at high frequencies may be approximated by $1 / f$. The broad, continuous frequency spectrum is a common feature of random fluctuations. ${ }^{22} \mathrm{Com}-$ parison of Figs. 9 and 8 indicates that the peak location of the power spectrum $f \approx 0.3 / t_{0} \approx 1.4 \mathrm{kHz}$ corresponds to the oscillation frequency of the correlation function, consistent with the Wiener-Khintchin theorem. ${ }^{23}$ In Fig. 9 a key difference between the frequency spectra is a relative lack of fluctuation power in the simulation at the low frequencies that are just below the peak value. Consequently, the spectral peak is narrower in the simulation than in the experiment.

Figure 10 shows the experimental and simulation data of the typical cross-correlation function in the vertical direction, defined as ${ }^{2} C(\Delta y, \tau)=\langle\widetilde{n}(y+\Delta y, t) \tilde{n}(y, t+\tau)\rangle / \sigma^{2}$, where $\langle\cdots\rangle$ indicates time average and $\Delta y$ is the vertical distance between the two signals. As seen in Fig. 10, the peak correlation amplitude is about 0.5 for the vertical distance $\Delta y \approx 5 \rho_{s} \approx 10 \mathrm{~cm}$ and has a negative time shift $\Delta \tau \approx 0.5 t_{0}$ $\approx 0.1 \mathrm{~ms}$ with respect to $\tau=0$. This indicates that the propagation of the instability measured in the laboratory frame is

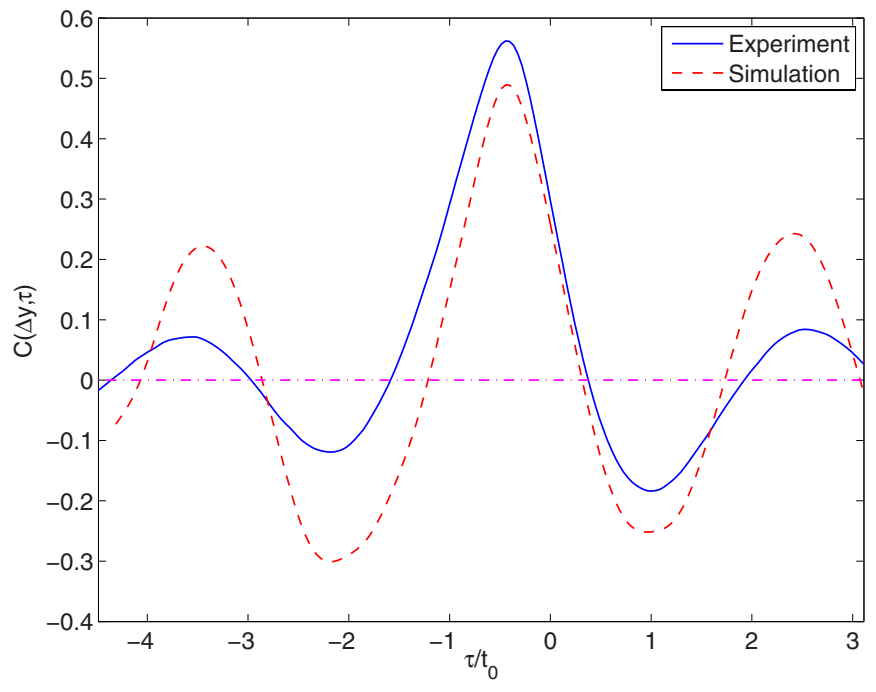

FIG. 10. (Color online) Comparison of experimental data (solid line) and simulation (dashed line) for the typical cross-correlation function in the vertical direction. Here $t_{0} \simeq 0.22 \mathrm{~ms}$.

upward (in the positive $y$ direction) and the propagation velocity $\Delta y / \Delta \tau \approx 0.2 c_{s} \approx 10^{3} \mathrm{~m} / \mathrm{s}$, which is about the local $\boldsymbol{E} \times \boldsymbol{B}$ velocity shown in Fig. 5 at $R \simeq 1.2 \mathrm{~m}$. Thus, the background $\boldsymbol{E} \times \boldsymbol{B}$ flow convects the interchange instability in the vertical direction.

The correlation functions in the radial direction have also been calculated. Measuring the decay of the peak correlation amplitude as a function of radial separation distance, we find that the radial correlation length $(\sim 1 / e$ decay length) $\lambda_{c} \simeq 4 \rho_{s} \approx 8 \mathrm{~cm}$, similar to the value observed in the experiment. ${ }^{2}$ The analysis of Ref. 4 predicts that the radial extent of interchange mode in this system is given by $\sqrt{L_{p} / k_{\Delta}} \approx 7 \rho_{s}$ for $L_{p} \simeq 13 \rho_{s}$ and $k_{\Delta} \rho_{s} \simeq 0.25$, which generally agrees with the radial correlation length in the simulation.

Figure 11 shows the experimental and simulation data of the typical PDF of the fluctuations, defined as $P_{j}=N_{j} / N h$, where $j$ is the bin number, $N_{j}$ is the count number in the $j$ th

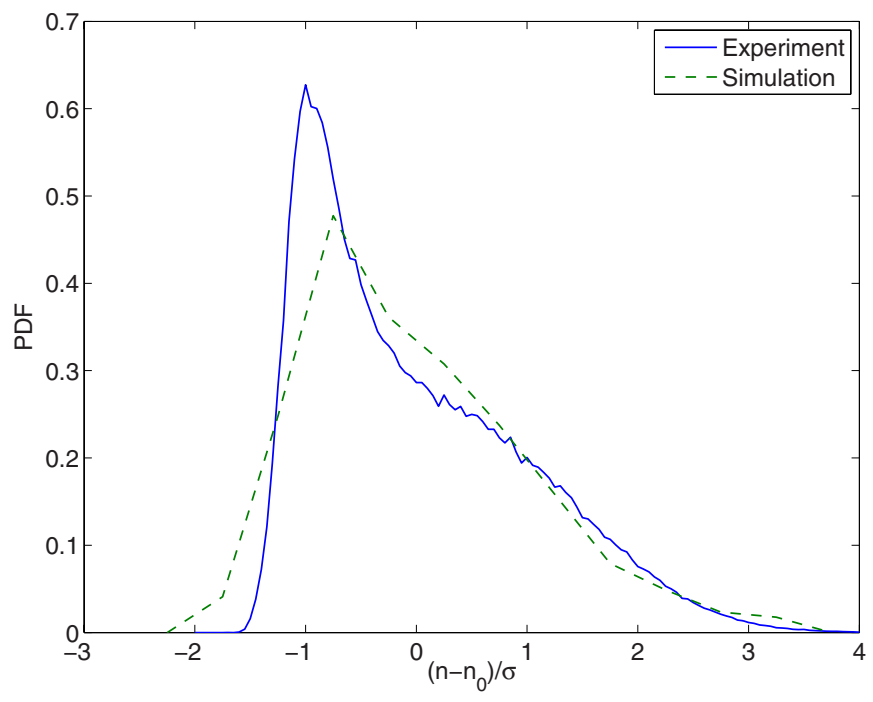

FIG. 11. (Color online) Comparison of experimental data (solid line) and simulation (dashed line) for the typical PDF of fluctuations. 
bin, $h$ is the bin width, and $N$ is the total number of data points. The fluctuation $\widetilde{n}(t)$ here is normalized by its standard deviation $\sigma$. The skewed distributions indicate that over a long period of time the low density events below the mean are predominant and the transport of high density plasma is intermittent. The calculations of the PDF at different radial positions show that the probability distributions are almost invariant on the low field side with similar non-Gaussian shapes. This indicates that the statistics of the fluctuations is nearly homogeneous in this region.

An apparent difference between the simulations and experiments not highlighted by the previous statistical measures concerns the fractional fluctuation levels, defined as the ratio of the standard deviation $\sigma$ of a given quantity to its mean value. In the experiment, the ion saturation current $I_{\text {sat }} \propto n \sqrt{T_{e}}$ is directly measured by Langmuir probes ${ }^{21}$ and yields $\tilde{I}_{\text {sat }} / I_{\text {sat }} \sim 0.4$ on the low field side. The corresponding deviations of $n \sqrt{T_{e}}$ in the simulations are about a factor of 3 smaller (0.14). The disagreement of fluctuation levels between experiment and simulation is also observed elsewhere. ${ }^{7}$ In the simulations, other fluctuation levels on the low field side are $\tilde{n} / n_{0} \sim 0.1, \widetilde{T}_{e} / T_{e 0} \sim 0.08$, and $\widetilde{\phi} / \phi_{0} \sim 0.1$. In general, the relative levels $\tilde{n} / n_{0}$ and $\widetilde{T}_{e} / T_{e 0}$ increase with $R$ and approach order unity values in the far edge (large $R$ ) region where the background values $n_{0}$ and $T_{e 0}$ are small. Since these background edge values are smaller in the experiments than in the simulations, as discussed earlier, this may account for the apparently larger relative fluctuation levels in the experiments. In addition, in contrast with the experiments, the modes with longer vertical wavelengths $k$ $<2 \pi / \Delta$ are strictly excluded in the simulations by the periodic boundary conditions. These longer-wavelength modes may also contribute to the quantitative differences between the simulation and experimental data.

\section{CONCLUSION}

In summary, we have studied the plasma transport and turbulence in the Helimak using a 2D fluid model that describes interchangelike fluctuations in a plasma with $\beta \ll 1$ and

$T_{i} \ll T_{e}$. The electrostatic two-fluid model evolves the full radial profiles of the plasma density, plasma potential, and electron temperature. The simulations include the $\boldsymbol{E} \times \boldsymbol{B}$ convection, magnetic curvature, plasma sources, and parallel losses and are found to be in good agreement with the analytic scalings obtained in Ref. 8. Focusing on the short connection length regime in an argon plasma, we find that the simulations capture many of the key features of experimental observations in the Helimak. An equilibrium plasma potential that produces sheared $\boldsymbol{E} \times \boldsymbol{B}$ flows is generated by sheath effects at the ends of open field lines. The comparisons of correlation functions and frequency spectra indicate that the toroidal return of the helical field lines introduces vertical periodicity on the $\Delta=2 \pi R B_{v} / B_{\varphi}$ scale, as one would expect for $k_{\|} \simeq 0$ fluctuations, and that the background $\boldsymbol{E} \times \boldsymbol{B}$ flow convects the interchangelike fluctuations in the vertical direction, as observed in the simulations. The power spectra of the fluctuations falloff like $1 / f$ at higher frequency. The comparisons of the PDF show that the skewed probability distributions on the low field side reflect the intermittent character of plasma transport.

\section{ACKNOWLEDGMENTS}

We are grateful to Amitava Bhattacharjee, Kevin Lee, Mario Podestà, Ivo Furno, and Brian LaBombard for helpful discussions.

This work was supported by Center for Integrated Computation and Analysis of Reconnection and Turbulence (CICART) under the auspices of the U.S. Department of Energy Experimental Program to Stimulate Competitive Research (EPSCoR) and DOE Grant Nos. DE-FG0207ER54915 and DE-FG02-04ER54766.

${ }^{1}$ K. W. Gentle and H. Huang, Plasma Sci. Technol. 10, 284 (2008).

${ }^{2}$ J. C. Perez, W. Horton, K. W. Gentle, W. L. Rowan, K. Lee, and R. B. Dahlburg, Phys. Plasmas 13, 032101 (2006).

${ }^{3}$ F. M. Poli, P. Ricci, A. Fasoli, and M. Podestà, Phys. Plasmas 15, 032104 (2008).

${ }^{4}$ P. Ricci, B. N. Rogers, and S. Brunner, Phys. Rev. Lett. 100, 225002 (2008).

${ }^{5}$ S. H. Müller, A. Fasoli, B. Labit, M. McGrath, M. Podestà, and F. Poli, Phys. Rev. Lett. 93, 165003 (2004).

${ }^{6}$ K. Rypdal and S. Ratynskaia, Phys. Rev. Lett. 94, 225002 (2005).

${ }^{7}$ P. Ricci, C. Theiler, A. Fasoli, I. Furno, B. Labit, S. H. Müller, M. Podestà, and F. M. Poli, Phys. Plasmas 16, 055703 (2009).

${ }^{8}$ P. Ricci and B. N. Rogers, Phys. Plasmas 16, 062303 (2009).

${ }^{9}$ A. Fasoli, B. Labit, M. McGrath, S. H. Müller, G. Plyushchev, M. Podestà, and F. M. Poli, Phys. Plasmas 13, 055902 (2006).

${ }^{10}$ A. Zeiler, J. F. Drake, and B. Rogers, Phys. Plasmas 4, 2134 (1997).

${ }^{11}$ O. E. Garcia, V. Naulin, A. H. Nielsen, and J. Juul Rasmussen, Phys. Plasmas 12, 062309 (2005).

${ }^{12}$ R. D. Hazeltine and F. L. Waelbroeck, The Framework of Plasma Physics (Perseus, Massachusetts, 1998).

${ }^{13}$ P. C. Stangeby, The Plasma Boundary of Magnetic Fusion Devices (Institute of Physics, Berkshire, 2000).

${ }^{14}$ O. E. Garcia, V. Naulin, A. H. Nielsen, and J. Juul Rasmussen, Phys. Rev. Lett. 92, 165003 (2004).

${ }^{15}$ N. Bisai, A. Das, S. Deshpande, R. Jha, P. Kaw, A. Sen, and R. Singh, Phys. Plasmas 11, 4018 (2004).

${ }^{16}$ D. A. D'Ippolito, J. R. Myra, and S. I. Krasheninnikov, Phys. Plasmas 9, $222(2002)$

${ }^{17}$ M. Podestà, A. Fasoli, B. Labit, M. McGrath, S. H. Müller, and F. M. Poli, Plasma Phys. Controlled Fusion 47, 1989 (2005).

${ }^{18}$ D. D. Ryutov and R. H. Cohen, Contrib. Plasma Phys. 44, 168 (2004).

${ }^{19}$ V. Naulin, J. Nycander, and J. Juul Rasmussen, Phys. Rev. Lett. 81, 4148 (1998).

${ }^{20}$ V. Naulin and A. H. Nielsen, SIAM J. Sci. Comput. (USA) 25, 104 (2003).

${ }^{21}$ M. A. Lieberman and A. J. Lichtenberg, Principles of Plasma Discharges and Materials Processing (Wiley, New York, 1994).

${ }^{22}$ B. Li, R. D. Hazeltine, and K. W. Gentle, Phys. Rev. E 76, 066402 (2007).

${ }^{23}$ B. Li and R. D. Hazeltine, Phys. Rev. E 73, 065402 (2006). 